PROCEEDINGS OF THE

AMERICAN MATHEMATICAL SOCIETY

Volume 125, Number 8, August 1997, Pages 2501-2502

S 0002-9939(97)03875-6

\title{
TWO POINT SET EXTENSIONS- A COUNTEREXAMPLE
}

\author{
JAN J. DIJKSTRA AND JAN VAN MILL
}

(Communicated by Franklin D. Tall)

\begin{abstract}
We show that there exist Cantor sets in the circle that are not extendable to sets that meet every line in the plane in exactly two points. This result solves a problem that was formulated by R. D. Mauldin.
\end{abstract}

A planar set is called a two point set if every line intersects the set in exactly two points and a partial two point set if every line intersects the set in at most two points. Circles and their subsets are obvious examples of partial two point sets. In [1] and [3], Problem 1070, Dan Mauldin asks the question whether every compact zero-dimensional partial two point set can be extended to a two point set. We show that the answer is no. Let $S^{1}$ stand for the unit circle in the plane centered at the origin $O$.

Proposition. There exists a Cantor set in $S^{1}$ that is not contained in a two point set.

Proof. Let $\lambda$ be the linear Lebesgue measure on smooth planar curves (lines and circles in our case). Select a dense open subset $U$ of $S^{1}$ such that $\lambda(U) \leq 1$ and $C=S^{1} \backslash U$ is a Cantor set. Let $x$ be a point in the plane with norm $|x| \geq 2$. Consider as in Figure 1 the two tangent lines to the circle through $x$. The tangent points $P$ and $Q$ divide $S^{1}$ into two open $\operatorname{arcs} A$ and $B$. The open line segment $L$ is perpendicular to the line through $x$ and $O$. Since $|x| \geq 2$ we have $\lambda(L) \geq 2 / \sqrt{3}>1$. Let $p_{A}$ and $p_{B}$ be the radial projections with respect to $x$ of $A$ respectively $B$ onto $L$. Note that both projections are contractions which implies that the image of each interval in $A$ or $B$ is an interval in $L$ of shorter length. This means that

$$
\begin{aligned}
\lambda\left(p_{A}(U \cap A) \cup p_{B}(U \cap B)\right) \leq \lambda\left(p_{A}(U \cap A)\right)+\lambda\left(p_{B}(U \cap B)\right) \\
\leq \lambda(U \cap A)+\lambda(U \cap B)=\lambda(U) \leq 1<\lambda(L) .
\end{aligned}
$$

If we pick a $y$ in $L \backslash\left(p_{A}(U \cap A) \cup p_{B}(U \cap B)\right)$ then the line through $x$ and $y$ intersects $C$ in two points.

Let $\ell$ be a line in the plane with distance at least 2 towards the origin. If $C$ is contained in a two point set $D$ then $D \cap \ell$ consists of two points. Pick an $x \in D \cap \ell$ and note that there is a line through $x$ that intersects $C$ in two points and hence it intersects $D$ in three points.

Received by the editors June 17, 1995 and, in revised form, February 29, 1996.

1991 Mathematics Subject Classification. Primary 54G20.

The first author is pleased to thank the Vrije Universiteit in Amsterdam for its hospitality and support. 


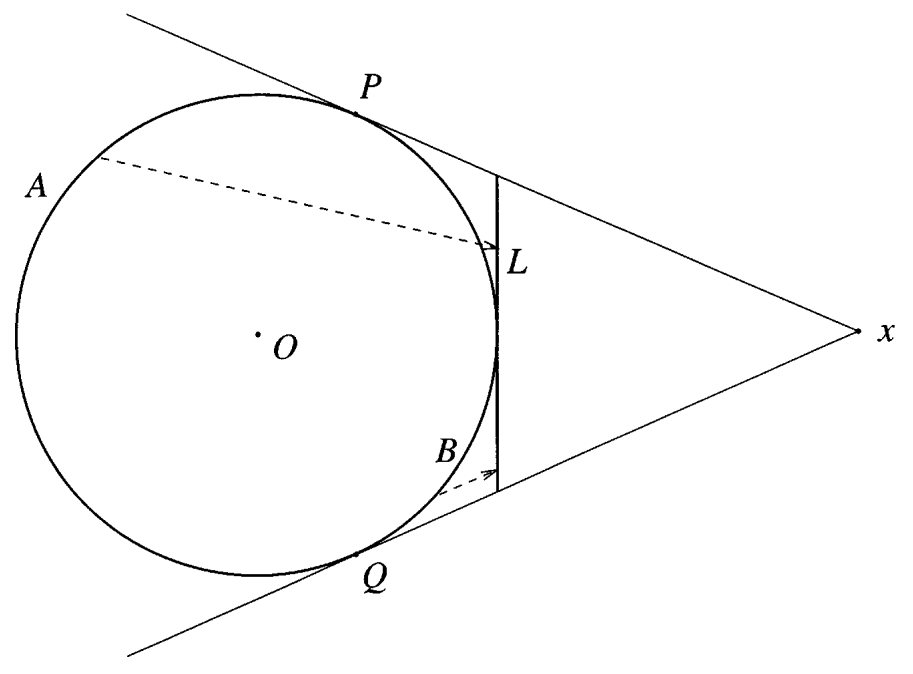

FiguRE 1

Remark. Observe that if $\lambda(U)$ approaches 0 then we can choose $x$ closer to the circle. In fact, we can show that if $C$ is a set in $S^{1}$ with $\lambda\left(S^{1} \backslash C\right) \leq 1$ then each point whose distance towards $S^{1}$ is at least $\lambda\left(S^{1} \backslash C\right)$ lies on a line that meets $C$ in two points. (For points $x$ inside the circle we apply a similar measure argument to the antipodal map $p_{x}: S^{1} \rightarrow S^{1}$ with respect to $x$, which has the property $\lambda\left(p_{x}(U)\right) \leq \frac{1+|x|}{1-|x|} \lambda(U)$.)

The referee informed us that our result also follows from a theorem that was announced by Dan Mauldin at the 1995 BEST conference in Boise, Idaho. Mauldin's theorem [2], which was obtained independently, is more general than our proposition.

\section{REFERENCES}

1. R. D. Mauldin, Problems in topology arising from analysis, Open Problems in Topology, J. van Mill and G. M. Reed, eds., North-Holland, Amsterdam, 1990, pp. 617-629. CMP 91:03

2. R. D. Mauldin, On sets which meet each line in exactly two points, in preparation.

3. J. van Mill and G. M. Reed, Open problems in topology, Topology Appl. 62 (1995), 93-99. MR 95m:54001

Department of Mathematics, The University of Alabama, Box 870350, Tuscaloosa, Alabama 35487-0350

E-mail address: jdijkstr@ua1vm.ua.edu

Faculteit Wiskunde en Informatica, Vrije Universiteit, De Boelelaan 1081A, 1081 HV Amsterdam, The Netherlands

E-mail address: vanmill@cs.vu.nl 